\title{
Stochastic processes driven by dichotomous Markov noise: Some exact dynamical results
}

\author{
J. M. Sancho \\ Departamento de Física Teórica, Facultad de Física, Diagonal, 647, Barcelona-28, Spain
}

(Received 29 September 1982; accepted for publication 13 January 1983)

\begin{abstract}
Stochastic processes defined by a general Langevin equation of motion where the noise is the nonGaussian dichotomous Markov noise are studied. A non-Fokker-Planck master differential equation is deduced for the probability density of these processes. Two different models are exactly solved. In the second one, a nonequilibrium bimodal distribution induced by the noise is observed for a critical value of its correlation time. Critical slowing down does not appear in this point but in another one.
\end{abstract}

PACS numbers: $05.40 .+\mathrm{j}, 02.50 .+\mathrm{s}, 02.30 . \mathrm{Jr}$

\section{INTRODUCTION}

Stochastic differential equations have an important and successful role in the theory of nonequilibrium phenomena. Most of them them are Langevin type, which are first-order differential equations with stochastic terms. In some cases the stochastic forces enter additively and often it is assumed that they represent internal fluctuations. In other cases the noise enters externally by means of a parameter of the phenomenological equation of motion which fluctuates. This kind of external noise has received a great deal of attention because it can represent a fluctuating external environment or a controlled noise generated in the laboratory by specific devices and introduced in the system in order to study its influence. This external noise is independent of the system and it is characterized by its intensity and correlation time. Examples of the influence of external noise can be found in a variety of systems, such as electric circuits' or liquid crystals, ${ }^{2}$ among others.

The mathematical study of these equations begins with the modeling of the noise. The simplest assumption is to take a Gaussian white noise which has zero correlation time. In this case, the process is Markovian and a Fokker-Planck equation for the probability density always exists. ${ }^{3}$ Nevertheless, this noise cannot always substitute for a real noise, which has a finite (perhaps small, but not zero) correlation time. In this case, the hypothesis of white noise, although suitable for a general description of the process, does not explore all the possibilities of a real noise. If we want to take into account the color of the noise, we should choose a mathematically tractable colored noise. Although many possible noises exist ${ }^{4}$ only two of them have been receiving enough consideration in the literature.

The first one is the Ornstein-Uhlenbeck process, which is Gaussian and obeys the same equation of motion as the velocity of a free Brownian particle. Stochastic processes driven by this noise have been studied in Refs. 5-8 and interesting features have been found which do not appear in the white noise assumption.

The second noise is the two-step Markov process or dichotomous noise. ${ }^{9,10}$ This noise is not Gaussian but Markovian and its influence in the stochastic process has been studied in Refs. 10-12. Interesting results, some of them quite similar to the former case, have been obtained, but only for the stationary state. A few dynamical properties are known in this case. ${ }^{12}$ This paper will be mainly devoted to the study of the dynamics of two stochastic processes which allow an exact analysis. These are linear models except for a change of variables, but they are not trivial. Moreover they present mainly the second example-characteristics belonging to the nonlinear cases, such as the possibility of having a nonequilibrium bimodal distribution.

The study is carried out by means of the evaluation of the first two moments of the stochastic variable and the solution of the differential equation that obeys the probability density.

Section 2 is devoted to the problem of finding the differential equation satisfied by the probability of the stochastic process. A short summary of the mathematical tools is presented and a differential equation of the non-Fokker-Planck type is obtained for the probability density. This new result is particularized to exact cases, whose probability density obeys a second-order partial differential equation of the hyperbolic type.

In Sec. 3 we study two exact examples: the first one is a pure diffusive model and the second one is a linear case with linear drift and additive noise. This last case is the most relevant and it will show interesting nonequilibrium characteristics. In Sec. 4 we summarize the main results of this paper.

\section{GENERAL THEORY}

\section{A. Differential equation for $P(q, t)$}

Here we summarize some known results. One can assume quite generally ${ }^{10.11}$ the following equation of motion for the variable $q$ :

$$
\dot{q}=f(q)+g(q) \xi(t)
$$

where $\xi(t)$ is a stochastic force that we identify with the dichotomous noise or two-step Markov process. ${ }^{13}$ This noise will only have two possible values $\pm \Delta$ with equal probability and jumps with probability $\frac{1}{2} \lambda d t$ for $d t .^{9}$ It has zero mean and autocorrelation

$$
\left\langle\xi(t) \xi\left(t^{\prime}\right)\right\rangle=\Delta^{2} \exp \left\{-\lambda\left|t-t^{\prime}\right|\right\}
$$

By means of the "formula of differentiation" of Shapiro 
and Loginov, ${ }^{14}$

$$
\begin{aligned}
& \frac{\partial}{\partial t}\langle\xi(t) \phi[\xi(t)]\rangle \\
& \quad=-\lambda\langle\xi(t) \phi[\xi(t)]\rangle+\left\langle\xi(t) \frac{\partial}{\partial t} \phi[\xi(t)]\right\rangle,
\end{aligned}
$$

where $\phi[\xi(t)]$ is a functional of $\xi(t)$ and the average is over the distribution of $\xi(t)$, we can obtain a closed set of equations for the probability density $P(q, t) .^{11,14}$ The method we are following $^{11}$ is an alternative to that employed by Kitahara et al. ${ }^{10}$ We begin with the stochastic Liouville equation ${ }^{15}$ for the density $\rho(q, t)$ of a set of realizations of $(2.1)$,

$$
\dot{\rho}(q, t)=-\frac{\partial}{\partial q}(f(q)+g(q) \xi(t)) \rho(q, t) .
$$

Taking the average over $\xi(t)$ and using Van Kampen's lemma ${ }^{9}$

$$
P(q, t)=\langle\rho(q, t)\rangle
$$

we arrive at

$$
\dot{P}(q, t)=-\frac{\partial}{\partial q} f(q) P(q, t)-\frac{\partial}{\partial q} g(q) P_{1}(q, t),
$$

where

$$
P_{1}(q, t)=\langle\xi(t) \rho(q, t)\rangle .
$$

Since $\rho(q, t)$ is a functional of $\xi(t)$, we will use the formula of differentiation (2.3) to obtain an equation of motion for $P_{1}(q, t)$ :

$$
\begin{aligned}
\dot{P}_{1}(q, t)= & -\lambda P_{1}(q, t)-\frac{\partial}{\partial q} f(q) P_{1}(q, t) \\
& -\frac{\partial}{\partial q} g(q) \Delta^{2} P(q, t),
\end{aligned}
$$

where we have used the fact that the square of the dichotomous noise is a constant $\xi^{2}(t)=\Delta^{2}$.

The set of equations (2.6) and (2.8) form a closed system of linear partial differential equations whose solution will give us $P(q, t)$, provided that we know the initial condition $P(q, 0)$. We also need another initial condition because we have two linear equations. The second one is obtained assuming statistical independence between $\xi(t)$ and $\rho(q, t)$ in $t=0^{14}$ :

$$
\left.\langle\xi(t) \rho(q, t)\rangle\right|_{t=0}=P_{1}(q, 0)=0,
$$

which implies in (2.6)

$$
\frac{\partial P(q, t)}{\partial t}-\left.\frac{\partial}{\partial q} f(q) P(q, t)\right|_{t=0}=0
$$

which together with

$$
\left.P(q, t)\right|_{t=0}=\delta(q)
$$

will be the initial conditions of the system (2.6), (2.8).

A closed equation for $P(q, t)$ cannot easily be obtained. Nevertheless, a formal expression can be given ${ }^{11}$ in the following way.

Let us formally integrate the linear equation (2.8):

$$
\begin{aligned}
P_{1}(q, t)= & -\Delta^{2} \int_{0}^{t} \exp \left\{-\left(\lambda+\frac{\partial}{\partial q} f(q)\right)\left(t-t^{\prime}\right)\right\} \\
& \frac{\partial}{\partial q} g(q) P\left(q, t^{\prime}\right) \equiv-\Delta^{2} B(q, t),
\end{aligned}
$$

where we have used (2.9). Substituting it in (2.6) we arrive at a formal differential equation for $P(q, t)^{11}$ :

$$
\dot{P}(q, t)=-\frac{\partial}{\partial q} f(q) P(q, t)+\Delta^{2} \frac{\partial}{\partial q} g(q) B(q, t) .
$$

The main object of this paper will be the exact solution, for two particular cases, of this integrodifferential equation.

Although the time-dependent solution is not always known, the stationary solution is well known and it reads, in the case that it exists, ${ }^{10,11}$

$$
\begin{aligned}
P_{\mathrm{st}}(q)= & N \frac{g(q)}{\Delta^{2} g^{2}(q)-f^{2}(q)} \\
& \times \exp \left\{\lambda \int^{q} \frac{d q^{\prime} f\left(q^{\prime}\right)}{\Delta^{2} g^{2}\left(q^{\prime}\right)-f^{2}\left(q^{\prime}\right)}\right\} .
\end{aligned}
$$

If in (2.13) we take

$$
\exp \left\{-\lambda\left|t-t^{\prime}\right|\right\} \simeq(2 / \lambda) \delta\left(t-t^{\prime}\right)
$$

we arrive at the white noise limit for $\xi(t)$. This limit holds for $\lambda \rightarrow \infty, \Delta \rightarrow \infty$, and $\Delta^{2} / \lambda$ finite and it give us an insight about a possible perturbation procedure, taking $\lambda$ as a large parameter.

\section{B. Expansion in $1 / \lambda$}

Let us review a perturbative approach to (2.13) considering $\lambda$ large. The limit $\lambda \rightarrow \infty$ is a very crude approximation because we lose all the specific characteristics of the dichotomous noise. Let us see how to retain the properties of $\xi(t)$ by means of an expansion in $1 / \lambda$.

We take a time derivative in (2.13)

$$
\begin{aligned}
\ddot{P}(q, t)= & -\frac{\partial}{\partial q} f(q) \dot{P}(q, t)+\Delta^{2} \frac{\partial}{\partial q} g(q) \frac{\partial}{\partial q} g(q) P(q, t) \\
& -\Delta^{2} \frac{\partial}{\partial q} g(q) \lambda\left(1+\frac{1}{\lambda} \frac{\partial}{\partial q} f(q)\right) B(q, t) .
\end{aligned}
$$

If we use now the approximation $(2.15)$ in $B(q, t)$, we obtain

$$
\begin{aligned}
\ddot{P}(q, t)= & -\frac{\partial}{\partial q} f(q) \dot{P}(q, t)+\Delta^{2} \frac{\partial}{\partial q} g(q) \frac{\partial}{\partial q} g(q) P(q, t) \\
& -\Delta^{2} \frac{\partial}{\partial q} g(q)\left(1+\frac{1}{\lambda} \frac{\partial}{\partial q} f(q)\right) \frac{\partial}{\partial q} g(q) P(q, t),
\end{aligned}
$$

which is valid to first order in $1 / \lambda$.

So we have reduced (2.13) to a second-order partial differential equation. The procedure is extended to the desired order in $1 / \lambda$, deriving (2.16) succesively.

At this moment, a question arises: does a process exist which obeys an equation exactly similar to (2.17)? The answer is affirmative and we are going to study it in the next subsection.

\section{Exact cases}

In (2.16) we can use (2.13) to substitute for the term $+\Delta^{2} \lambda(\partial g(q) / \partial q) B(q, t)$

$$
\Delta^{2} \frac{\partial}{\partial q} g(q) B(q, t)=\dot{P}(q, t)+\frac{\partial}{\partial q} f(q) P(q, t) .
$$

The other term $-\Delta^{2}(\partial g(q) / \partial q)(\partial f(q) / \partial q) B(q, t)$ needs a careful analysis. By means of the commutation of the $q$ derivatives, this term is expressed as (Note: from now on an 
upper point means a partial time-derivative and a comma means a $q$-derivative):

$$
\begin{aligned}
-\Delta^{2} & \frac{\partial}{\partial q} g(q) \frac{\partial}{\partial q} f(q) B(q, t) \\
= & -\Delta^{2} \frac{\partial}{\partial t}\left(g(q) f^{\prime}(q)-g^{\prime}(q) f(q)\right) B(q, t) \\
& -\Delta^{2} \frac{\partial}{\partial q} f(q) \frac{\partial}{\partial q} g(q) B(q, t),
\end{aligned}
$$

where the last term can be written in terms of $P(q, t)$ by means of $(2.13)$ so that

$$
\begin{aligned}
-\Delta^{2} & \frac{\partial}{\partial q} f(g) \frac{\partial}{\partial q} g(q) B(q, t) \\
& =-\frac{\partial}{\partial q} f(q) \dot{P}(q, t)-\frac{\partial}{\partial q} f(q) \frac{\partial}{\partial q} f(g) P(q, t) .
\end{aligned}
$$

Joining all these partial results, the gereral equation (2.16) can be written in the following form:

$$
\begin{aligned}
\ddot{P}(q, t)= & -\lambda \dot{P}(q, t)-2 \frac{\partial}{\partial q} f(q) \dot{P}(q, t) \\
& -\lambda \frac{\partial}{\partial q} f(q) P(q, t) \\
& +\Delta^{2} \frac{\partial}{\partial q} g(q) \frac{\partial}{\partial q} g(q) P(q, t) \\
& -\frac{\partial}{\partial q} f(q) \frac{\partial}{\partial q} f(q) P(q, t) \\
& -\frac{\partial}{\partial q}\left(g(q) f^{\prime}(q)-g^{\prime}(q) f(q)\right) \Delta^{2} B(q, t) .
\end{aligned}
$$

As far as solvability is concerned, this formal equation is equivalent to (2.16). In order to advance in this way, we need to eliminate $B(q, t)$ in the last term in $(2.21)$. This can be done in the case that

$$
g(q) f^{\prime}(q)-g^{\prime}(q) f(q)=g^{2}(q)(f(q) / g(q))^{\prime}=C g(q),
$$

where $C$ is a constant. If this condition holds, the last term in $(2.21)$ is $-\partial(C g(q) B(q, t)) / \partial q$ and by means of $(2.13)$, it is transformed into

$$
\begin{aligned}
-C & \frac{\partial}{\partial q} g(q) \Delta{ }^{2} B(q, t) \\
& =-C P(q, t)-C \frac{\partial}{\partial q} f(q) P(q, t),
\end{aligned}
$$

and hence $(2.21)$

$$
\begin{aligned}
\ddot{P}(q, t)= & -(\lambda+C) \dot{P}(q, t)-2 \frac{\partial}{\partial q}(q) \dot{P}(q, t) \\
& -(\lambda+C) \frac{\partial}{\partial q} f(q) P(q, t) \\
& +\Delta^{2} \frac{\partial}{\partial q} g(q) \frac{\partial}{\partial q} g(q) P(q, t) \\
& -\frac{\partial}{\partial q} f(q) \frac{\partial}{\partial q} f(q) P(q, t),
\end{aligned}
$$

which is a second-order partial differential equation for the probability density of the process (2.1). This equation is one of the main results of this paper and it has a time-dependent exact solution with the initial conditions (2.10) and (2.11). For this reason, those processes (2.1) obeying (2.22) will be called exactly solvable models and not surprisingly the con- dition (2.22) is the same one that was discussed by Hänggi ${ }^{16}$ and San Miguel ${ }^{17}$ in the context of the white noise hypothesis for the stochastic force $\xi(t)$ in (2.1) and by Sancho and San Miguel $^{5}$ in the case of a Gaussian but a nonwhite assumption for $\xi(t)$.

In all these cases, the equation for the probability density was of first order in time (Fokker-Planck equation) with a linear drift and a constant diffusion whose solution is well known. In our case, there are higher time derivatives and the exact solution is, as of now, unknown. The exact solution of (2.24) will be another important result of this paper.

Before starting with the process of solving (2.24), we will write it in the standard form of second-order partial differential equations. This is done by commuting the $q$-derivatives

$$
\begin{aligned}
& {\left[\frac{\partial}{\partial t^{2}}+\left(f^{2}(q)-\Delta^{2} g(q)\right) \frac{\partial^{2}}{\partial q^{2}}\right.} \\
& \quad+2 f(q) \frac{\partial^{2}}{\partial t \partial q}+\left(\lambda+C+2 f^{\prime}(q)\right) \frac{\partial}{\partial t} \\
& \quad+\left((\lambda+C) f(q)-3 \Delta^{2} g(q) g^{\prime}(q)+3 f(q) f^{\prime}(q)\right) \frac{\partial}{\partial q} \\
& \quad+\left(\left(f(q) f^{\prime}(q)\right)^{\prime}-\Delta^{2}\left(g(q) g^{\prime}(q)\right)^{\prime}\right. \\
& \left.\left.\quad+(\lambda+C) f^{\prime}(q)\right)\right] P(q, t) .
\end{aligned}
$$

In order to classify this partial differential equation, we need to evaluate the discriminant, which is

$$
\left(\Delta^{2} g^{2}(q)\right)^{1 / 2}=\Delta g(q) \geqslant 0
$$

because $g(q)$ should always be positive. Equation(2.25) is classified as an hyperbolic second-order partial differential equation for whose solution we are going to follow the current studies on this mathematical topic.

\section{EXAMPLES}

If our process $(2.1)$ obeys the necessary and sufficient condition $(2.22)$ to be exactly solvable, we define a new variable $Q(q(t)), 5,16,17$

$$
Q(q)=\int^{q} \frac{d q}{g(q)},
$$

and we have

$$
\dot{Q}=f(q) / g(q)+\xi(t)=C Q+A+\xi(t),
$$

where we have used (2.22) in the integrated form and $A$ is an irrelevant integration constant. We can assume $A$ equal to zero. Our problem has been reduced to a linear one with additive noise. This is well known $n^{5,16,17}$ in the context of soluble cases.

Equation (3.2) with $\boldsymbol{A}=0$ presents two possible and different versions: $C$ equal to zero or not. These two cases will be called the pure diffusive case and the linear case, respectively.

\section{A. Pure diffusive case}

This case corresponds to the equation of motion (2.1) with $f(q)=0$. The representative model can be written, after performing the changes (3.1) and relabeling the variable as

$$
\dot{q}(t)=\xi(t) \text {. }
$$


Some exact results can be obtained without solving the corresponding equation of motion for $P(q, t)$. For example, the statistical averages are obtained as follows: The solution of (3.3) is

$$
q(t)=q(0)+\int_{0}^{t} \xi\left(t^{\prime}\right) d t^{\prime}
$$

and hence the first two moments of the variable $q$ are

$$
\begin{aligned}
\langle q(t)\rangle=\langle q(0)\rangle_{\mathrm{IC}} & =0, \\
\left\langle q^{2}(t)\right\rangle-\left\langle q^{2}(0)\right\rangle_{\mathrm{IC}} & =\int_{0}^{t} \xi(s) d s \int_{0}^{t} \xi\left(s^{\prime}\right) d s^{\prime} \\
& =\frac{2 \Delta^{2}}{\lambda}\left(t-\frac{\left(1-e^{-\lambda t}\right)}{\lambda}\right) .
\end{aligned}
$$

They are expressed in terms of the initial averages and we have used the statistical properties of $\xi(t)$ in (2.2). These results coincide with the well-known ones for the position of a free Brownian particle. We can obtain more interesting information about the system obeying (3.3). The knowledge of the time-dependent probability density $P(q, t)$ shows interesting behavior very different from that in the white noise limit.

From the general expression (2.25), particularized to the model $(3.3), f(q)=0, g(q)=1$, we obtain the equation of motion for $P(q, t)$ :

$$
\frac{\partial^{2}}{\partial t^{2}} P(q, t)-\Delta^{2} \frac{\partial^{2}}{\partial q^{2}} P(q, t)+\lambda \frac{\partial}{\partial t} P(q, t)=0 .
$$

The initial conditions $(2.10)$ and (2.11) become in this case

$$
\begin{aligned}
& \left.P(q, t)\right|_{t=0}=\delta(q), \\
& \left.\frac{\partial P(q, t)}{\partial t}\right|_{t=0}=0 .
\end{aligned}
$$

A similar equation to (3.6) with the initial conditions (3.7) and (3.8) appeared in the context of the generalized Smoluchowski diffusion equations ${ }^{18.19}$ and the present example was solved by Hemmer. ${ }^{20}$ Therefore, we will not reproduce the details. The probability density is

$$
\begin{aligned}
P(q, t)= & \frac{1}{2} e^{-\lambda t / 2}[\delta(\Delta t-q)+\delta(\Delta t+q)] \\
& +\frac{\lambda}{2 \Delta} I_{0}\left(\frac{\lambda}{4 \Delta}\left(\Delta^{2} t^{2}-q^{2}\right)^{1 / 2}\right) \\
& +\frac{\lambda t}{2\left(\Delta^{2} t^{2}-q^{2}\right)^{1 / 2}} I_{1}\left(\frac{\lambda}{2 \Delta}\left(\Delta^{2} t^{2}-q^{2}\right)^{1 / 2}\right),
\end{aligned}
$$

where $I_{0}, I_{1}$ are the Bessel functions of the imaginary arguments of orders 0,1 , respectively.

From (3.9) one can see that $P(q, t)$ is almost a flat distribution bounded by two delta functions moving to $q= \pm \infty$ with a velocity $\pm \Delta$, respectively. This shape is very different from that corresponding to the white noise case, ${ }^{20}$ which presents a Gaussian distribution spreading out in time.

\section{B. Linear case}

In this case, Eq. (2.1) takes the general form (3.2), and after relabeling the variables, it is expressed as

$$
\dot{q}=-\gamma q+\xi(t) .
$$

As in the former case, some interesting results can be obtained using the formal solution of $(3.10)$. This is

$$
q(t)=q(0) e^{-r t}+\int_{0}^{t} e^{-x^{t}-t^{\prime}} \xi\left(t^{\prime}\right) d t^{\prime},
$$

so the mean value is

$$
\langle q(t)\rangle=\langle q(0)\rangle_{\mathrm{IC}} e^{-r t},
$$

which goes to zero for $t \rightarrow \infty$.

An interesting dynamical quantity is the correlation function

$$
\left\langle q(t) q\left(t^{\prime}\right)\right\rangle .
$$

Using the solution (3.11), the statistical properties of $\xi(t)$ in (2.2), and assuming statistical independence between the initial conditions and $\xi(t)$, with $q(0)=0$, the quantity (3.13) is

$$
\begin{aligned}
\left\langle q(t) q\left(t^{\prime}\right)\right\rangle= & \frac{2 \Delta^{2}}{\lambda+\gamma}\left(\frac{1}{\gamma-\lambda}-\frac{1}{2 \gamma}\right) e^{-\gamma^{\left(t+t^{\prime}\right)}} \\
& -\frac{\Delta^{2}}{\gamma^{2}-\lambda^{2}} e^{-\gamma t-\lambda t^{\prime}}-\frac{\Delta^{2}}{\gamma^{2}-\lambda^{2}} e^{-\gamma t^{\prime}-\lambda t} \\
& +\left(\frac{\Delta^{2}}{(\lambda+\gamma) \gamma}-\frac{\Delta^{2}}{\gamma^{2}-\lambda^{2}}\right) e^{-\gamma^{\left(t-t^{\prime}\right)}} \\
& +\frac{\Delta^{2}}{\gamma^{2}-\lambda^{2}} e^{-\lambda\left(t-t^{\prime}\right)}
\end{aligned}
$$

and in the stationary state $\left(t, t^{\prime} \rightarrow \infty\right)$ but $t-t^{\prime}$ finite,

$$
\begin{aligned}
\left\langle q(t) q\left(t^{\prime}\right)\right\rangle_{\mathrm{st}}= & -\frac{\lambda \Delta^{2}}{\gamma\left(\gamma^{2}-\lambda^{2}\right)} e^{-\gamma\left(t-t^{\prime}\right)} \\
& +\frac{\Delta^{2}}{\gamma^{2}-\lambda^{2}} e^{-\lambda\left(t-t^{\prime}\right)} .
\end{aligned}
$$

The equal-time correlation function in the stationary state is

$$
\left\langle q^{2}\right\rangle_{\mathrm{st}}=\Delta^{2} / \gamma(\lambda+\gamma) .
$$

From the exact solution (3.15), we can obtain the linear relaxation time and see if critical slowing down exists at any point:

$$
\tau=\int_{0}^{\infty} \frac{\left\langle q(t) q\left(t+t^{\prime}\right\rangle_{\mathrm{st}}\right.}{\left\langle q^{2}(t)\right\rangle_{\mathrm{st}}} d t^{\prime}=\frac{\lambda+\gamma}{\lambda \gamma} .
$$

We can see that only in the cases $\gamma \rightarrow 0$ or $\lambda \rightarrow 0$, the linear relaxation time diverges. In both cases, the formal stationary probability density is not normalizable, as we will see. The first case $(\gamma \rightarrow 0)$ is a trivial one because the dissipative drift disappears and the problem reduces to the case $3 \mathrm{~A}$. The second case $(\lambda \rightarrow 0)$ is new and more interesting because even with nonzero dissipative drift, the "color" $\lambda$ and not the intensity $\Delta$ of the noise $\xi(t)$ precludes the existence of a stationary state. This corresponds to having a noise with infinite correlation time, and hence in the opposite limit of white noise.

The stationary distribution can be obtained from (2.14):

$$
P_{\mathrm{st}}(q)=N\left(\Delta^{2}-\gamma^{2} q^{2}\right)^{\lambda / 2 \gamma-1},
$$

defined between the boundaries $q= \pm \Delta / \gamma$. The normalization constant is

$$
N=\frac{\gamma \Gamma\left(\frac{1}{2}+\lambda / 2 \gamma\right)}{\Delta^{\lambda / \gamma-1} \Gamma\left(\frac{1}{2}\right) \Gamma(\lambda / 2 \gamma)} .
$$

One can see that the correlation function in the stationary state evaluated with (3.18) and (3.19) agrees with (3.16).

The study of (3.18) manifests two different shapes for $P_{\text {st }}(q)$, which we relate to a nonequilibrium phase transition. 
The critical value of the parameters is $\lambda=2 \gamma$. In the case $\lambda>2 \gamma, P_{\mathrm{st}}(q)$ has a maximum in $q=0$ and goes to zero in the boundaries. For $\lambda<2 \gamma, P_{\mathrm{st}}(q)$ has a minimum in $q=0$ and goes to infinity at the boundaries. This gives rise to a bimodal distribution when we change the "color" of the noise $\lambda$ from $\lambda>2 \gamma$ to $\lambda<2 \gamma$. Then we have found a nonequilibrium phase transition induced only by the "color" of the noise. The transition takes place at the value $\lambda=2 \gamma$. We have seen in (3.17) that no critical slowing down appears in this point but in $\lambda \rightarrow 0$. This is a very interesting example of Suzuki's theory, ${ }^{8}$ which states that in nonequilibrium phase transitions, the phenomena of critical slowing down and the appearance of new maxima in $P_{\mathrm{st}}(q)$ are separate processes which can take place for different values of the parameters.

We should mention that in the case $\lambda \rightarrow \infty$, we recover the white noise $P_{\text {st }}(q)$ corresponding to this problem. Let us now return to the problem of finding the solution of $P(q, t)$, which in this case is not known. As the details of the mathematical process are very cumbersome, we will state only the main steps and references used in the evaluation.

In the model (3.10), where $f(q)=-\gamma q, g(q)=1$, the equation of motion $(2.25)$ for the probability density is

$$
\begin{gathered}
{\left[\frac{\partial^{2}}{\partial t^{2}}+\left(\gamma^{2} q^{2}-\Delta^{2}\right) \frac{\partial^{2}}{\partial q^{2}}-2 \gamma q \frac{\partial^{2}}{\partial t \partial q}\right.} \\
-(3 \gamma-\lambda) \frac{\partial}{\partial t}-\gamma(\lambda-4 \gamma) q \\
\left.\quad \times \frac{\partial}{\partial q}-\gamma(\lambda-2 \gamma)\right] P(q, t)=0
\end{gathered}
$$

with the corresponding initial conditions (2.10) and (2.11):

$$
\begin{aligned}
& \left.P(q, t)\right|_{t=0}=\delta(q), \\
& \left.\left(\frac{\partial}{\partial t}-\gamma q \frac{\partial}{\partial q}-\gamma\right) P(q, t)\right|_{t=0}=0 .
\end{aligned}
$$

The standard approach in the solution of $(3.20)^{21}$ begins with its reduction to the canonical form. This is done by means of a change of variables

$$
\begin{aligned}
& \xi=e^{\gamma t}(\gamma q-\Delta), \\
& \eta=e^{\gamma t}(\gamma q+\Delta),
\end{aligned}
$$

where the new variables $\xi, \eta$ are called the characteristics. The partial differential equation for $P(\xi, \eta)$ takes the hyperbolic form

$$
\begin{aligned}
& {\left[(\eta-\xi)^{2} \frac{\partial^{2}}{\partial \xi \partial \eta}-\alpha(\eta-\xi) \frac{\partial}{\partial \xi}\right.} \\
& \left.\quad+\alpha(\eta-\xi) \frac{\partial}{\partial \eta}-2 \alpha\right] P(\xi, \eta)=0,
\end{aligned}
$$

where

$$
\alpha=1-\lambda / 2 \gamma .
$$

The equation (3.25) has been studied by Koshlyakov et al. ${ }^{21}$ in several cases. We follow their approach. The next step is to transform (3.25) into the Euler-Darbouse equation by the change

$$
P(\xi, \eta)=(\eta-\xi)^{\beta} Q(\xi, \eta) .
$$

$Q(\xi, \eta)$ obeys the partial differential equation

$$
\left[\frac{\partial^{2}}{\partial \xi \partial \eta}+\frac{\alpha}{\eta-\xi} \frac{\partial}{\partial \xi}-\frac{\alpha}{\eta-\xi} \frac{\partial}{\partial \eta}\right] Q(\xi, \eta)=0
$$

with

$$
\beta=2 \alpha .
$$

The solution of the Cauchy problem associated with (3.28) with the corresponding boundary conditions given by (3.21) and (3.22), following the Rieman method, is indicated in Ref. 21. This gives, after transforming variables,

$$
P(q, t)=P_{1}(q, t)+P_{2}(q, t)+P_{3}(q, t),
$$

where

$$
\begin{aligned}
& P_{1}(q, t)= \frac{1}{2} \gamma e^{(\alpha-1) \gamma^{\prime}}\left\{\delta\left(\Delta\left(1-e^{-\gamma t}\right)+\gamma q\right)+\delta\left(\gamma q-\Delta\left(q-e^{-\gamma t}\right)\right)\right\}, \\
& P_{2}(q, t)= \gamma(1-2 \alpha)(2 \Delta)^{2 \alpha-1}\left(\Delta^{2}\left(1+e^{-\gamma t}\right)^{2}-\gamma^{2} q^{2}\right)^{-\alpha} F(\alpha, \alpha, 1 ; \sigma), \\
& P_{3}(q, t)=-\frac{1}{2} \gamma(2 \Delta)^{2 \alpha+1} e^{-\gamma t}\left(\gamma q-\Delta\left(1+e^{-\gamma t}\right)\right)^{-1}\left(\Delta^{2}\left(1+e^{-\gamma t}\right)^{2}-\gamma^{2} q^{2}\right)^{-\alpha} F(\alpha, \alpha, 1 ; \sigma) \\
& \quad+\frac{1}{2} \gamma(2 \Delta)^{2 \alpha+1} e^{-\gamma t}\left(\Delta^{2}\left(1+e^{-\gamma^{t}}\right)^{2}-\gamma^{2} q^{2}\right)^{-\alpha} F^{\prime}(\alpha, \alpha, 1 ; \sigma)\left(\gamma^{2} g^{2}-\Delta^{2}\left(1+e^{-\gamma^{t}}\right)^{2}\right)^{-1} \\
& \quad \times\left\{\left(\gamma q+\Delta\left(1-e^{-\gamma t}\right)\right)\left(\gamma q+\Delta\left(1+e^{-\gamma^{t}}\right)\right)^{-1}+\left(\gamma q-\Delta\left(1-e^{-\gamma^{t}}\right)\right)\left(\gamma q-\Delta\left(1+e^{-\gamma^{t}}\right)\right)^{-1}\right\}, \\
& \sigma=\left(\Delta^{2}\left(1-e^{-\gamma^{t}}\right)^{2}-\gamma^{2} q^{2}\right)\left(\Delta^{2}\left(1+e^{-\gamma^{t}}\right)^{2}-\gamma^{2} q^{2}\right)^{-1}, \\
&|q| \leqslant(\Delta / \gamma)\left(1 \pm e^{-\gamma^{t}}\right) .
\end{aligned}
$$

$F(\alpha, \alpha, 1 ; \sigma)$ and $F^{\prime}(\alpha, \alpha, 1 ; \sigma)$ are the hypergeometric function and its derivative with respect to $\sigma$.

$P_{1}(q, t)$ gives the behavior of $P(q, t)$ in the initial regime as one can see taking the limit $t \rightarrow 0 . P_{2}(q, t)$ dominates in the limit $t \rightarrow \infty$, giving the stationary solution $P_{\mathrm{st}}(q)$, which coincides with (3.18) and (3.19). $P_{3}(q, t)$ refers to the intermediate regime.

Although the solution(3.30)-(3.35) has its own importance because of its existence, only a few results can be obtained from it because of its extraordinary complexity. This shows that the non-Gaussianity of the process (3.10) manifests itself by the complexity of the solution, even in the case that we have a linear problem. As in the former case, we have that $P(q, t)$ is bounded by two delta functions moving to the stationary boundaries $\pm \Delta / \gamma$, and following a deterministic equation given by

$$
\dot{q}_{ \pm}=-\gamma q_{ \pm} \pm \Delta \quad \text { or } \quad \dot{q}_{ \pm}=f\left(q_{ \pm}\right) \pm g\left(q_{ \pm}\right) \Delta,
$$


where $q_{ \pm}$is the peak position of the delta functions. This is easily understood if we think that the stochastic dichotomous process has only two possible values $\pm \Delta$. This is a characteristic of this kind of stochastic process modeled by this special noise, which has also been found in nonlinear equations by means of numerical simulation, and whose results will be presented elsewhere.

\section{CONCLUSIONS}

We have explored the possibility of obtaining dynamical properties for a Langevin-like equation of motion with dichotomous Markov noise. We have presented a general method to obtain the differential equation obeyed by the probability density of the process. This differential equation involves higher time derivatives and hence it is not of the Fokker-Planck type. The main point in this deduction is to consider the correlation time of the noise as an expansion parameter. In two particular cases, we have been able to write an exact differential equation which is a second-order partial differential equation of the hyperbolic type. In these two cases, we have found the exact solution of $P(q, t)$. In the second case, which has a nontrivial stationary state, we have studied the possibility of the appearance of critical slowing down by means of the explicit evaluation of the correlation time. Although the stationary analysis gives the existence of a phase transition for some value of the noise parameters, no critical slowing down appears in this point but in another one. This is an example of Suzuki's criteria of the appearance of slowing down in nonequilibrium stochastic processes.

\section{ACKNOWLEDGMENT}

I am indebted to Dr. M. San Miguel for his valuable comments.

'S. Kabashima, S. Kogure, S. Kawakubo, and T. Okada, J. Appl. Phys. 50, 6296 (1979).

${ }^{2}$ S. Kai, T. Kai, M. Takata, and K. Hirakawa, J. Phys. Soc. Jpn. 47, 1379 (1979).

${ }^{3}$ R. L. Stratonovich, Topics in the Theory of Random Noise, Vol. 1 (Gordon and Breach, New York, 1963).

${ }^{4}$ A. Brissaud and U. Frisch, J. Math. Phys. 15, 524 (1974).

5J. M. Sancho and M. San Miguel, Z. Phys. B 36, 357 (1980).

${ }^{6} \mathrm{M}$. San Miguel and J. M. Sancho, Phys. Lett. A 76, 97 (1980).

${ }^{7}$ W. Horsthemke and R. Lefever, Z. Phys. B 40, 241 (1980).

${ }^{8}$ M. Suzuki, K. Kaneko, and F. Sasagawa, Progr. Theoret. Phys. 65, 828 (1981).

${ }^{9}$ N. G. Van Kampen, Phys. Rep. 24, 171 (1976).

${ }^{10}$ K. Kitahara, W. Horsthemke, and R. Lefever, Phys. Lett. A 70, 377 (1979); K. Kitahara, W. Horsthemke, R. Lefever, and Y. Inaba, Progr Theoret. Phys. 64, 1233 (1980).

${ }^{1}$ J J. M. Sancho and M. San Miguel, Progr. Theoret. Phys. (1983) (to be published).

${ }^{12} \mathrm{M}$. Suzuki, in Systems far from Equilibrium, edited by L. Garrido, Lecture Notes in Physics, Vol. 132 (Springer-Verlag, Berlin, 1980); K. Kitahara and $\mathrm{K}$. Ishii, "Relaxation of systems under the influence of two level Markovian noise," oral communication in Statphys 14 (Edmonton, Canada, 1981); C. Van den Broeck, Phys. Lett. A 91, 399 (1982).

${ }^{13}$ R. C. Bourret, U. Frisch, and A. Pouquet, Physica 65, 303 (1973).

${ }^{14}$ V. E. Shapiro and W. M. Loginov, Physica A 91, 563 (1978).

${ }^{15}$ R. Kubo, J. Math. Phys. 4, 174 (1963); M. Suzuki, Progr. Theoret. Phys. Suppl. 69, 169 (1980).

${ }^{16}$ P. Hänggi, Z. Phys. B 30, 85 (1978).

${ }^{17}$ M. San Miguel, Z. Phys. B 33, 307 (1979).

${ }^{18}$ H. C. Brinkman, Physica 22, $29(1956)$.

${ }^{19}$ R. A. Sack, Physica 22, 917 (1956).

${ }^{20}$ P. Chr. Hemmer, Physica 27, 79 (1961).

${ }^{21}$ N. S. Koshlyakov, M. M. Smirnov, and R. R. Gliner, in Differential Equations of Mathematical Physics (North-Holland, Amsterdam, 1964). 